\title{
Two-Component Super AKNS Equations and Their Finite-Dimensional Integrable Super Hamiltonian System
}

\author{
Jing $\mathrm{Yu}^{1}$ and Jingwei Han \\ ${ }^{1}$ School of Science, Hangzhou Dianzi University, Hangzhou, Zhejiang 310018, China \\ ${ }^{2}$ School of Information Engineering, Hangzhou Dianzi University, Hangzhou, Zhejiang 310018, China \\ Correspondence should be addressed to Jingwei Han; jingweih@hdu.edu.cn
}

Received 29 December 2013; Accepted 4 March 2014; Published 31 March 2014

Academic Editor: Weiguo Rui

Copyright (c) $2014 \mathrm{~J}$. Yu and J. Han. This is an open access article distributed under the Creative Commons Attribution License, which permits unrestricted use, distribution, and reproduction in any medium, provided the original work is properly cited.

Starting from a matrix Lie superalgebra, two-component super AKNS system is constructed. By making use of monononlinearization technique of Lax pairs, we find that the obtained two-component super AKNS system is a finite-dimensional integrable super Hamiltonian system. And its Lax representation and $r$-matrix are also given in this paper.

\section{Introduction}

The inverse scattering method provides us with a powerful tool to generate multicomponent soliton equations. In [14], they have constructed many multicomponent soliton equations, which are much more important for physicists and mathematicians than one-component ones, owing to the fact that they possess rich structure and have more extensive prospect.

Monononlinearization of Lax pair is a method to obtain finite-dimensional integrable Hamiltonian system, which was firstly proposed by Cao in [5]. The main idea of monononlinearization includes the following three aspects. Firstly, they find a symmetry constraint between potential and eigenfunctions. Secondly, substituting the symmetry constraint into the spectral problem, they obtain constrained finite-dimensional system. Lastly, they show that obtained constrained system is Hamiltonian system and completely integrable in the Liouville sense. Many finite-dimensional integrable Hamiltonian systems are constructed in [6-10]. This method was generalized by Ma and Strampp in [11]. The main difference of binary-nonlinearization and monononlinearization lies in the following two aspects. One is that binary-nonlinearization needs to introduce adjoint spectral problem, and, thus, both spectral problem and adjoint spectral problem constitute even-dimensional system. The other is that symmetry constraint proposed in the procedure of binary-nonlinearization is not only associated with eigenfunctions but also associated with adjoint eigenfunctions. According to the Liouville integrable theorem which states that a $2 n$-dimensional Hamiltonian system over some region $\Omega \in R^{2 n}$ with $n$ independent integrals of motion in involution may be integrated by quadratures, we cannot apply monononlinearization to odd-dimensional spectral problem. However, we can apply both monononlinearization and binary-nonlinearization to the even-dimensional spectral problem. Many finite-dimensional integrable Hamiltonian systems are constructed in [12-14]. Owing to one-component super integrable system which is associated with a $3 \times 3$ spectral matrix, we just consider binary-nonlinearization in our previous papers [15-17]. From the above analysis, we propose the following questions. Does even-dimensional spectral problem of super integrable system exist? If it exists, can we apply monononlinearization to the super integrable system? All of these questions will be answered in this paper.

The paper is organized as follows. In Section 2, we derive two-component super AKNS system and write this new system as the super Hamiltonian form. In Section 3, we find a symmetry constraint which is only associated with eigenfunctions, and, after substituting the symmetry constraint into the $N$ copies spectral problem, we obtained $6 N$ dimensional constrained system. Furthermore, we rewrite 
the $6 \mathrm{~N}$-dimensional system as the super Hamiltonian form. Lax representation and $r$-matrix of the constrained system are given in Section 4. In the last section, some conclusions and discussions are given.

\section{Two-Component Super AKNS System}

Let us start with the following linear space $G=\left\{e_{1}, e_{2}, e_{3}\right.$, $\left.e_{4}, e_{5}\right\}$ :

$$
\begin{gathered}
e_{1}=\left(\begin{array}{ccc}
E & 0 & 0 \\
0 & -E & 0 \\
0 & 0 & 0
\end{array}\right), \quad e_{2}=\left(\begin{array}{ccc}
0 & E & 0 \\
0 & 0 & 0 \\
0 & 0 & 0
\end{array}\right), \\
e_{3}=\left(\begin{array}{lll}
0 & 0 & 0 \\
E & 0 & 0 \\
0 & 0 & 0
\end{array}\right), \quad e_{4}=\left(\begin{array}{ccc}
0 & 0 & E \\
0 & 0 & 0 \\
0 & -E & 0
\end{array}\right), \\
e_{5}=\left(\begin{array}{lll}
0 & 0 & 0 \\
0 & 0 & E \\
E & 0 & 0
\end{array}\right),
\end{gathered}
$$

where $E=\operatorname{diag}(1,1)$ is a $2 \times 2$ unit matrix, 0 is a $2 \times 2$ zero matrix, $G_{0}=\left\{e_{1}, e_{2}, e_{3}\right\}$ is even, and $G_{1}=\left\{e_{4}, e_{5}\right\}$ is odd. After a direct calculation, we obtain that

$$
\begin{array}{rlr}
{\left[e_{1}, e_{2}\right\}=2 e_{2},} & {\left[e_{1}, e_{3}\right\}=-2 e_{3},} & {\left[e_{2}, e_{3}\right\}=e_{1},} \\
{\left[e_{1}, e_{4}\right\}=e_{4},} & {\left[e_{1}, e_{5}\right\}=-e_{5},} & {\left[e_{2}, e_{5}\right\}=e_{4},} \\
{\left[e_{3}, e_{4}\right\}=e_{5},} & {\left[e_{4}, e_{4}\right\}=-2 e_{2},} & {\left[e_{4}, e_{5}\right\}=e_{1},} \\
{\left[e_{5}, e_{5}\right\}=2 e_{3},} &
\end{array}
$$

and the others are zeros, where

$$
[a, b\}=a b-(-1)^{p(a) p(b)} b a
$$

is the super Lie bracket and $p(f)$ denotes the parity of the arbitrary element $f$.

It is easy to prove that the linear space $G$ is matrix Lie superalgebra. The corresponding loop superalgebra $\widetilde{G}$ is presented as

$$
\begin{gathered}
e_{i}(n)=e_{i} \lambda^{n}, \\
{\left[e_{1}(m), e_{2}(n)\right\}=2 e_{2}(m+n),} \\
{\left[e_{1}(m), e_{3}(n)\right\}=-2 e_{3}(m+n),} \\
{\left[e_{2}(m), e_{3}(n)\right\}=e_{1}(m+n),} \\
{\left[e_{1}(m), e_{4}(n)\right\}=e_{4}(m+n),} \\
{\left[e_{1}(m), e_{5}(n)\right\}=-e_{5}(m+n),} \\
{\left[e_{2}(m), e_{4}(n)\right\}=\left[e_{3}(m), e_{5}(n)\right\}=0,} \\
{\left[e_{2}(m), e_{5}(n)\right\}=e_{4}(m+n),} \\
{\left[e_{3}(m), e_{4}(n)\right\}=e_{5}(m+n),} \\
{\left[e_{4}(m), e_{4}(n)\right\}=-2 e_{2}(m+n),}
\end{gathered}
$$

$$
\begin{array}{r}
{\left[e_{4}(m), e_{5}(n)\right\}=e_{1}(m+n),} \\
{\left[e_{5}(m), e_{5}(n)\right\}=2 e_{3}(m+n),} \\
\operatorname{deg} e_{i}(n)=n, \quad(i=1,2,3,4,5) .
\end{array}
$$

In what follows, we will construct multicomponent super integrable equations via the matrix Lie superalgebra $G$. Firstly, let us consider the following superisospectral problem:

$$
\phi_{x}=U(u, \lambda) \phi
$$

where

$$
U(u, \lambda)=-e_{1}(1)+q e_{2}(0)+r e_{3}(0)+\alpha e_{4}(0)+\beta e_{5}(0),
$$

which can be written as the following matrix form:

$$
\phi_{x}=\left(\begin{array}{ccc}
-\lambda E & q & \alpha \\
r & \lambda E & \beta \\
\beta & -\alpha & 0
\end{array}\right) \phi,
$$

where $q=\operatorname{diag}\left(q_{1}, q_{2}\right), r=\operatorname{diag}\left(r_{1}, r_{2}\right), \alpha=\operatorname{diag}\left(\alpha_{1}, \alpha_{2}\right)$, $\beta=\operatorname{diag}\left(\beta_{1}, \beta_{2}\right), u=(q, r, \alpha, \beta)^{T}$ is a potential, $\lambda$ is a spectral parameter which satisfies $\lambda_{t_{n}}=0$, and $\phi=\left(\phi_{1}, \ldots, \phi_{6}\right)^{T}$ is an eigenfunction.

Taking

$$
V=\left(\begin{array}{ccc}
a & b & \rho \\
c & -a & \delta \\
\delta & -\rho & 0
\end{array}\right),
$$

where $a=\operatorname{diag}\left(a_{1}, a_{2}\right), b=\operatorname{diag}\left(b_{1}, b_{2}\right), c=\operatorname{diag}\left(c_{1}, c_{2}\right), \rho=$ $\operatorname{diag}\left(\rho_{1}, \rho_{2}\right)$, and $\delta=\operatorname{diag}\left(\delta_{1}, \delta_{2}\right)$, the adjoint representation equation

$$
V_{x}=[U, V]
$$

leads to

$$
\begin{gathered}
a_{k, x}=q_{k} c_{k}-r_{k} b_{k}+\alpha_{k} \delta_{k}+\beta_{k} \rho_{k}, \quad k=1,2, \\
b_{k, x}=-2 \lambda b_{k}-2 q_{k} a_{k}-2 \alpha_{k} \rho_{k}, \quad k=1,2, \\
c_{k, x}=2 \lambda c_{k}+2 r_{k} a_{k}+2 \beta_{k} \delta_{k}, \quad k=1,2, \\
\rho_{k, x}=-\lambda \rho_{k}+q_{k} \delta_{k}-\alpha_{k} a_{k}-\beta_{k} b_{k}, \quad k=1,2, \\
\delta_{k, x}=\lambda \delta_{k}+r_{k} \rho_{k}-\alpha_{k} c_{k}+\beta_{k} a_{k}, \quad k=1,2 .
\end{gathered}
$$

On setting $a_{k}=\sum_{m \geq 0} a_{k}^{(m)} \lambda^{-m}, b_{k}=\sum_{m \geq 0} b_{k}^{(m)} \lambda^{-m}, c_{k}=$ $\sum_{m \geq 0} c_{k}^{(m)} \lambda^{-m}, \rho_{k}=\sum_{m \geq 0} \rho_{k}^{(m)} \lambda^{-m}$, and $\delta_{k}=\sum_{m \geq 0} \delta_{k}^{(m)} \lambda^{-m}$ $(k=1,2)$, (10) engenders equivalently

$$
\begin{gathered}
b_{k}^{(0)}=c_{k}^{(0)}=\rho_{k}^{(0)}=\delta_{k}^{(0)}=0, \quad k=1,2, \\
a_{k, x}^{(m)}=q_{k} c_{k}^{(m)}-r_{k} b_{k}^{(m)}+\alpha_{k} \delta_{k}^{(m)}+\beta_{k} \rho_{k}^{(m)}, \quad k=1,2, m \geq 0, \\
b_{k, x}^{(m)}=-2 b_{k}^{(m+1)}-2 q_{k} a_{k}^{(m)}-2 \alpha_{k} \rho_{k}^{(m)}, \quad k=1,2, m \geq 0, \\
c_{k, x}^{(m)}=2 c_{k}^{(m+1)}+2 r_{k} a_{k}^{(m)}+2 \beta_{k} \delta_{k}^{(m)}, \quad k=1,2, m \geq 0,
\end{gathered}
$$




$$
\begin{array}{cc}
\rho_{k, x}^{(m)}=-\rho_{k}^{(m+1)}+q_{k} \delta_{k}^{(m)}-\alpha_{k} a_{k}^{(m)}-\beta_{k} b_{k}^{(m)}, & L_{42}=\operatorname{diag}\left(2 \beta_{1}-2 \alpha_{1} \partial^{-1} r_{1}, 2 \beta_{2}-2 \alpha_{2} \partial^{-1} r_{2}\right), \\
k=1,2, m \geq 0, & L_{43}=\operatorname{diag}\left(-q_{1}+\alpha_{1} \partial^{-1} \alpha_{1},-q_{2}+\alpha_{2} \partial^{-1} \alpha_{2}\right), \\
\delta_{k, x}^{(m)}=\delta_{k}^{(m+1)}+r_{k} \rho_{k}^{(m)}-\alpha_{k} c_{k}^{(m)}+\beta_{k} a_{k}^{(m)}, \quad k=1,2, m \geq 0, & L_{44}=\operatorname{diag}\left(-\partial-\alpha_{1} \partial^{-1} \beta_{1},-\partial-\alpha_{2} \partial^{-1} \beta_{2}\right) .
\end{array}
$$

which results in a recursion relation to determine $a_{k}^{(m)}, b_{k}^{(m)}$, $c_{k}^{(m)}, \rho_{k}^{(m)}, \delta_{k}^{(m)}(k=1,2)$ :

$$
\left(\begin{array}{cc}
c_{1}^{(m+1)} & c_{2}^{(m+1)} \\
b_{1}^{(m+1)} & b_{2}^{(m+1)} \\
2 \delta_{1}^{(m+1)} & 2 \delta_{2}^{(m+1)} \\
-2 \rho_{1}^{(m+1)} & -2 \rho_{2}^{(m+1)}
\end{array}\right)=L\left(\begin{array}{cc}
c_{1}^{(m)} & c_{2}^{(m)} \\
b_{1}^{(m)} & b_{2}^{(m)} \\
2 \delta_{1}^{(m)} & \\
-2 \rho_{1}^{(m)} & 2 \delta_{2}^{(m)} \\
& -2 \rho_{2}^{(m)}
\end{array}\right),
$$

where

$$
L=\left(\begin{array}{llll}
L_{11} & L_{12} & L_{13} & L_{14} \\
L_{21} & L_{22} & L_{23} & L_{24} \\
L_{31} & L_{32} & L_{33} & L_{34} \\
L_{41} & L_{42} & L_{43} & L_{44}
\end{array}\right)
$$

with

$$
\begin{aligned}
& L_{11}=\operatorname{diag}\left(\frac{1}{2} \partial-r_{1} \partial^{-1} q_{1}, \frac{1}{2} \partial-r_{2} \partial^{-1} q_{2}\right), \\
& L_{12}=\operatorname{diag}\left(r_{1} \partial^{-1} r_{1}, r_{2} \partial^{-1} r_{2}\right) \\
& L_{13}=\operatorname{diag}\left(-\frac{1}{2} r_{1} \partial^{-1} \alpha_{1}-\frac{1}{2} \beta_{1},-\frac{1}{2} r_{2} \partial^{-1} \alpha_{2}-\frac{1}{2} \beta_{2}\right), \\
& L_{14}=\operatorname{diag}\left(\frac{1}{2} r_{1} \partial^{-1} \beta_{1}, \frac{1}{2} r_{2} \partial^{-1} \beta_{2}\right) \\
& L_{21}=\operatorname{diag}\left(-q_{1} \partial^{-1} q_{1},-q_{2} \partial^{-1} q_{2}\right) \\
& L_{22}=\operatorname{diag}\left(-\frac{1}{2} \partial+q_{1} \partial^{-1} r_{1},-\frac{1}{2} \partial+q_{2} \partial^{-1} r_{2}\right), \\
& L_{23}=\operatorname{diag}\left(-\frac{1}{2} q_{1} \partial^{-1} \alpha_{1},-\frac{1}{2} q_{2} \partial^{-1} \alpha_{2}\right) \\
& L_{24}=\operatorname{diag}\left(\frac{1}{2} \alpha_{1}+\frac{1}{2} q_{1} \partial^{-1} \beta_{1}, \frac{1}{2} \alpha_{2}+\frac{1}{2} q_{2} \partial^{-1} \beta_{2}\right), \\
& L_{31}=\operatorname{diag}\left(2 \alpha_{1}-2 \beta_{1} \partial^{-1} q_{1}, 2 \alpha_{2}-2 \beta_{2} \partial^{-1} q_{2}\right), \\
& L_{32}=\operatorname{diag}\left(2 \beta_{1} \partial^{-1} r_{1}, 2 \beta_{2} \partial^{-1} r_{2}\right), \\
& L_{33}=\operatorname{diag}\left(\partial-\beta_{1} \partial^{-1} \alpha_{1}, \partial-\beta_{2} \partial^{-1} \alpha_{2}\right), \\
& L_{34}=\operatorname{diag}\left(r_{1}+\beta_{1} \partial^{-1} \beta_{1}, r_{2}+\beta_{2} \partial^{-1} \beta_{2}\right), \\
& L_{41}=\operatorname{diag}\left(2 \alpha_{1} \partial^{-1} q_{1}, 2 \alpha_{2} \partial^{-1} q_{2}\right),
\end{aligned}
$$

It is easy to obtain that $a_{k, x}^{(0)}=0(k=1,2)$. Therefore, we choose $a_{k}^{(0)}=-1(k=1,2)$ and select constants of integral to be zero, which lead to the first few terms being worked out as follows:

$$
\begin{gathered}
a_{k}^{(1)}=0, \quad b_{k}^{(1)}=q_{k}, \quad c_{k}^{(1)}=r_{k}, \\
\rho_{k}^{(1)}=\alpha_{k}, \quad \delta_{k}^{(1)}=\beta_{k}, \\
a_{k}^{(2)}=\frac{1}{2} q_{k} r_{k}+\alpha_{k} \beta_{k}, \quad b_{k}^{(2)}=-\frac{1}{2} q_{k, x}, \quad c_{k}^{(2)}=\frac{1}{2} r_{k, x},
\end{gathered}
$$$$
\rho_{k}^{(2)}=-\alpha_{k, x}, \quad \delta_{k}^{(2)}=\beta_{k, x}
$$

where $k=1,2$.

Secondly, let us associate (5) with the following auxiliary spectral problem:

$$
\phi_{t_{n}}=V^{(n)} \phi
$$

where

$$
V^{(n)}=\sum_{m=0}^{n}\left(\begin{array}{cccccc}
a_{1}^{(m)} & 0 & b_{1}^{(m)} & 0 & \rho_{1}^{(m)} & 0 \\
0 & a_{2}^{(m)} & 0 & b_{2}^{(m)} & 0 & \rho_{2}^{(m)} \\
c_{1}^{(m)} & 0 & -a_{1}^{(m)} & 0 & \delta_{1}^{(m)} & 0 \\
0 & c_{2}^{(m)} & 0 & -a_{2}^{(m)} & 0 & \delta_{2}^{(m)} \\
\delta_{1}^{(m)} & 0 & -\rho_{1}^{(m)} & 0 & 0 & 0 \\
0 & \delta_{2}^{(m)} & 0 & -\rho_{2}^{(m)} & 0 & 0
\end{array}\right) \lambda^{n-m}
$$

The compatibility condition of (5) and (16) leads to the famous zero curvature equations

$$
U_{t_{n}}-V_{x}^{(n)}+\left[U, V^{(n)}\right]=0, \quad n \geq 1,
$$

which lead to isospectral super integrable equations

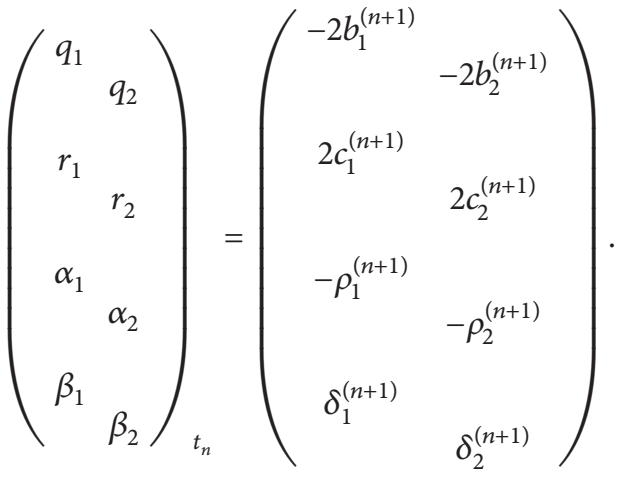


Under the special reduction of $q_{2}=r_{2}=\alpha_{2}=\delta_{2}=0$, (19) is equivalent to the super AKNS equations [15, 18, 19], and thus (19) is called a two-component super AKNS equation.

Lastly, super Hamiltonian structures of the twocomponent super AKNS equations (19) may be established by applying a powerful tool, that is, the so-called supertrace identity $[20,21]$

$$
\frac{\delta}{\delta u} \int \operatorname{str}\left(V \frac{\partial U}{\partial \lambda}\right) d x=\lambda^{-\gamma} \frac{\partial}{\partial \lambda} \lambda^{\gamma} \operatorname{str}\left(\frac{\partial U}{\partial u} V\right)
$$

As is usual, we need the following equalities which are easy to calculate:

$$
\begin{gathered}
\operatorname{str}\left(V \frac{\partial U}{\partial \lambda}\right)=-2\left(a_{1}+a_{2}\right), \quad \operatorname{str}\left(\frac{\partial U}{\partial q_{k}} V\right)=c_{k}, \\
\operatorname{str}\left(\frac{\partial U}{\partial r_{k}} V\right)=b_{k}, \quad \operatorname{str}\left(\frac{\partial U}{\partial \alpha_{k}} V\right)=2 \delta_{k}, \\
\operatorname{str}\left(\frac{\partial U}{\partial \beta_{k}} V\right)=-2 \rho_{k},
\end{gathered}
$$

where $k=1,2$. Substituting the above equality (21) into the supertrace identity (20) and comparing the coefficients of $\lambda^{-m-2}$ on two sides, we arrive at

$$
\left.\left(\begin{array}{cc}
\frac{\delta}{\delta q_{1}} & \\
& \frac{\delta}{\delta q_{2}} \\
\frac{\delta}{\delta r_{1}} & \\
\frac{\delta}{\delta \alpha_{1}} & \frac{\delta}{\delta r_{2}} \\
\frac{\delta}{\delta \beta_{1}} & \frac{\delta}{\delta \alpha_{2}} \\
=(\gamma-m-1) & \frac{\delta}{\delta \beta_{2}}
\end{array}\right) \begin{array}{cc}
-2\left(a_{1}^{(m+2)}+a_{2}^{(m+2)}\right) d x \\
c_{1}^{(m+1)} & c_{2}^{(m+1)} \\
2 \delta_{1}^{(m+1)} & b_{2}^{(m+1)} \\
-2 \rho_{1}^{(m+1)} & 2 \delta_{2}^{(m+1)} \\
& -2 \rho_{2}^{(m+1)}
\end{array}\right),
$$

which leads to the constant $\gamma=0$ with $m=0$. Thus, we have

$$
\left.\begin{array}{cc}
c_{1}^{(m+1)} & \\
b_{1}^{(m+1)} & c_{2}^{(m+1)} \\
2 \delta_{1}^{(m+1)} & b_{2}^{(m+1)} \\
-2 \rho_{1}^{(m+1)} & 2 \delta_{2}^{(m+1)} \\
-2 \rho_{2}^{(m+1)}
\end{array}\right)=\left(\begin{array}{cc}
\frac{\delta}{\delta q_{1}} & \\
\frac{\delta}{\delta r_{1}} & \frac{\delta}{\delta q_{2}} \\
\frac{\delta}{\delta \alpha_{1}} & \frac{\delta}{\delta r_{2}} \\
\frac{\delta}{\delta \beta_{1}} & \frac{\delta}{\delta \alpha_{2}} \\
\bar{H}_{m}= & =\frac{2}{m+2}\left(a_{1}^{(m+2)}+a_{2}^{(m+2)}\right) d x .
\end{array}\right.
$$

Now, it follows from (23) that the two-component super AKNS system (19) has the following super bi-Hamiltonian structure:

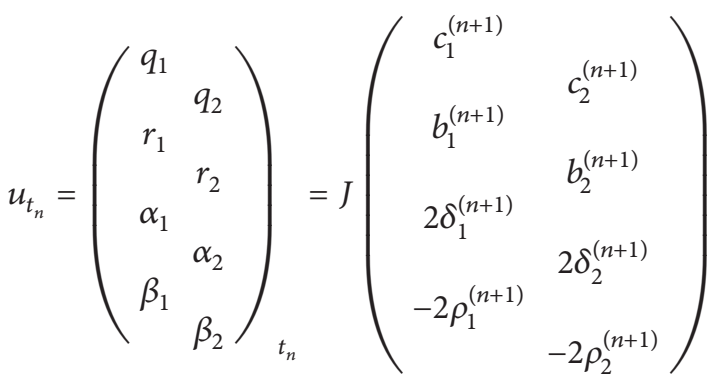

$$
\begin{aligned}
& =J \frac{\delta \bar{H}_{n}}{\delta u}=M \frac{\delta \bar{H}_{n-1}}{\delta u},
\end{aligned}
$$

where the super Hamiltonian pair $(J, M=J L)$ reads as

$$
\begin{gathered}
J=\left(\begin{array}{cccc}
0 & -2 E & 0 & 0 \\
2 E & 0 & 0 & 0 \\
0 & 0 & 0 & \frac{1}{2} E \\
0 & 0 & \frac{1}{2} E & 0
\end{array}\right), \\
M=J L=\left(\begin{array}{cccc}
-2 L_{21} & -2 L_{22} & -2 L_{23} & -2 L_{24} \\
2 L_{11} & 2 L_{12} & 2 L_{13} & 2 L_{14} \\
\frac{1}{2} L_{41} & \frac{1}{2} L_{42} & \frac{1}{2} L_{43} & \frac{1}{2} L_{44} \\
\frac{1}{2} L_{31} & \frac{1}{2} L_{32} & \frac{1}{2} L_{33} & \frac{1}{2} L_{34}
\end{array}\right),
\end{gathered}
$$

where $L_{m n}(1 \leq m, n \leq 4)$ are given by (13). 
The first nonlinear equations in two-component AKNS system (24) are given by

$$
\begin{aligned}
& q_{1, t_{2}}=-\frac{1}{2} q_{1, x x}+q_{1}^{2} r_{1}+2 q_{1} \alpha_{1} \beta_{1}-2 \alpha_{1} \alpha_{1, x}, \\
& q_{2, t_{2}}=-\frac{1}{2} q_{2, x x}+q_{2}^{2} r_{2}+2 q_{2} \alpha_{2} \beta_{2}-2 \alpha_{2} \alpha_{2, x}, \\
& r_{1, t_{2}}=\frac{1}{2} r_{1, x x}-q_{1} r_{1}^{2}-2 r_{1} \alpha_{1} \beta_{1}-2 \beta_{1} \beta_{1, x}, \\
& r_{2, t_{2}}=\frac{1}{2} r_{2, x x}-q_{2} r_{2}^{2}-2 r_{2} \alpha_{2} \beta_{2}-2 \beta_{2} \beta_{2, x}, \\
& \alpha_{1, t_{2}}=-\alpha_{1, x x}-q_{1} \beta_{1, x}+\frac{1}{2} q_{1} r_{1} \alpha_{1}-\frac{1}{2} q_{1, x} \beta_{1},
\end{aligned}
$$

$$
\begin{aligned}
& \alpha_{2, t_{2}}=-\alpha_{2, x x}-q_{2} \beta_{2, x}+\frac{1}{2} q_{2} r_{2} \alpha_{2}-\frac{1}{2} q_{2, x} \beta_{2}, \\
& \beta_{1, t_{2}}=\beta_{1, x x}+r_{1} \alpha_{1, x}+\frac{1}{2} r_{1, x} \alpha_{1}-\frac{1}{2} q_{1} r_{1} \beta_{1}, \\
& \beta_{2, t_{2}}=\beta_{2, x x}+r_{2} \alpha_{2, x}+\frac{1}{2} r_{2, x} \alpha_{2}-\frac{1}{2} q_{2} r_{2} \beta_{2},
\end{aligned}
$$

and the corresponding temporal part of the Lax system is

$$
\phi_{t_{2}}=V^{(2)} \phi
$$

where

$$
V^{(2)}=\left(\begin{array}{cccccc}
-\lambda^{2}+v_{1} & 0 & q_{1} \lambda-\frac{1}{2} q_{1, x} & 0 & \alpha_{1} \lambda-\alpha_{1, x} & 0 \\
0 & -\lambda^{2}+v_{2} & 0 & q_{2} \lambda-\frac{1}{2} q_{2, x} & 0 & \alpha_{2} \lambda-\alpha_{2, x} \\
r_{1} \lambda+\frac{1}{2} r_{1, x} & 0 & \lambda^{2}-v_{1} & 0 & \beta_{1} \lambda+\beta_{1, x} & 0 \\
0 & r_{2} \lambda+\frac{1}{2} r_{2, x} & 0 & \lambda^{2}-v_{2} & 0 & \beta_{2} \lambda+\beta_{2, x} \\
\beta_{1} \lambda+\beta_{1, x} & 0 & -\alpha_{1} \lambda+\alpha_{1, x} & 0 & 0 & 0 \\
0 & \beta_{2} \lambda+\beta_{2, x} & 0 & -\alpha_{2} \lambda+\alpha_{2, x} & 0 & 0
\end{array}\right),
$$

with $v_{k}=(1 / 2) q_{k} r_{k}+\alpha_{k} \beta_{k}(k=1,2)$.

\section{Finite-Dimensional Super Hamiltonian System}

In this section, we will apply monononlinearization to the two-component super AKNS system (24). It is easy to find that, for the super spectral problem (1), variational derivative of $\lambda$ with respect to the potential $u$ reads (up to a constant factor)

$$
\frac{\delta \lambda}{\delta u}=\left(\begin{array}{cc}
\frac{\delta \lambda}{\delta q_{1}} & \\
\frac{\delta \lambda}{\delta r_{1}} & \frac{\delta \lambda}{\delta q_{2}} \\
\frac{\delta \lambda}{\delta \alpha_{1}} & \frac{\delta \lambda}{\delta r_{2}} \\
\frac{\delta \lambda}{\delta \beta_{1}} & \frac{\delta \lambda}{\delta \alpha_{2}} \\
& \frac{\delta \beta_{2}}{\delta \beta_{2}}
\end{array}\right)=\left(\begin{array}{cc}
\phi_{3}^{2} & \phi_{4}^{2} \\
-\phi_{1}^{2} & -\phi_{2}^{2} \\
2 \phi_{3} \phi_{5} & 2 \phi_{4} \phi_{6} \\
-2 \phi_{1} \phi_{5} & -2 \phi_{2} \phi_{6}
\end{array}\right)
$$

When zero boundary conditions $\left(\lim _{|x| \rightarrow+\infty} \phi_{j}=0,1 \leq j \leq\right.$ 6 ) are imposed, we can verify a simple characteristic property of the above variational derivative:

$$
L \frac{\delta \lambda}{\delta u}=\lambda \frac{\delta \lambda}{\delta u}
$$

where $L$ is given by (13).
To carry out nonlinearization of $N$ copies of systems (7) and (27) with $N$ distinct parameters $\lambda_{j}(1 \leq j \leq N)$, we consider the following Bargmann symmetry constraint:

$$
\begin{array}{cc}
q_{1}=-\left\langle\Phi_{1}, \Phi_{1}\right\rangle, & q_{2}=-\left\langle\Phi_{2}, \Phi_{2}\right\rangle, \\
r_{1}=\left\langle\Phi_{3}, \Phi_{3}\right\rangle, & r_{2}=\left\langle\Phi_{4}, \Phi_{4}\right\rangle, \\
\alpha_{1}=\left\langle\Phi_{1}, \Phi_{5}\right\rangle, & \alpha_{2}=\left\langle\Phi_{2}, \Phi_{6}\right\rangle, \\
\beta_{1}=\left\langle\Phi_{3}, \Phi_{5}\right\rangle, & \beta_{2}=\left\langle\Phi_{4}, \Phi_{6}\right\rangle,
\end{array}
$$

where $\Phi_{j}=\left(\phi_{j 1}, \ldots, \phi_{j N}\right)^{T}(1 \leq j \leq 6)$ and $\langle\cdot, \cdot\rangle$ refers to the standard inner product of the Euclidian space $\mathbb{R}^{N}$. Now, substituting the constraint (31) into $N$ copies of system (7), we obtain the following constrained vector system:

$$
\begin{aligned}
& \Phi_{1, x}=-\Lambda \Phi_{1}-\left\langle\Phi_{1}, \Phi_{1}\right\rangle \Phi_{3}+\left\langle\Phi_{1}, \Phi_{5}\right\rangle \Phi_{5}=\frac{\partial H_{1}}{\partial \Phi_{3}}, \\
& \Phi_{2, x}=-\Lambda \Phi_{2}-\left\langle\Phi_{2}, \Phi_{2}\right\rangle \Phi_{4}+\left\langle\Phi_{2}, \Phi_{6}\right\rangle \Phi_{6}=\frac{\partial H_{1}}{\partial \Phi_{4}}, \\
& \Phi_{3, x}=\left\langle\Phi_{3}, \Phi_{3}\right\rangle \Phi_{1}+\Lambda \Phi_{3}+\left\langle\Phi_{3}, \Phi_{5}\right\rangle \Phi_{5}=-\frac{\partial H_{1}}{\partial \Phi_{1}}, \\
& \Phi_{4, x}=\left\langle\Phi_{4}, \Phi_{4}\right\rangle \Phi_{2}+\Lambda \Phi_{4}+\left\langle\Phi_{4}, \Phi_{6}\right\rangle \Phi_{6}=-\frac{\partial H_{1}}{\partial \Phi_{2}},
\end{aligned}
$$




$$
\begin{aligned}
& \Phi_{5, x}=\left\langle\Phi_{3}, \Phi_{5}\right\rangle \Phi_{1}-\left\langle\Phi_{1}, \Phi_{5}\right\rangle \Phi_{3}=\frac{\partial H_{1}}{\partial \Phi_{5}}, \\
& \Phi_{6, x}=\left\langle\Phi_{4}, \Phi_{6}\right\rangle \Phi_{2}-\left\langle\Phi_{2}, \Phi_{6}\right\rangle \Phi_{4}=\frac{\partial H_{1}}{\partial \Phi_{6}},
\end{aligned}
$$

where

$$
\begin{aligned}
H_{1}= & -\left\langle\Lambda \Phi_{1}, \Phi_{3}\right\rangle-\left\langle\Lambda \Phi_{2}, \Phi_{4}\right\rangle-\frac{1}{2}\left\langle\Phi_{1}, \Phi_{1}\right\rangle\left\langle\Phi_{3}, \Phi_{3}\right\rangle \\
& -\frac{1}{2}\left\langle\Phi_{2}, \Phi_{2}\right\rangle\left\langle\Phi_{4}, \Phi_{4}\right\rangle+\left\langle\Phi_{1}, \Phi_{5}\right\rangle\left\langle\Phi_{3}, \Phi_{5}\right\rangle \\
& +\left\langle\Phi_{2}, \Phi_{6}\right\rangle\left\langle\Phi_{4}, \Phi_{6}\right\rangle,
\end{aligned}
$$

with $\Lambda=\operatorname{diag}\left(\lambda_{1}, \ldots, \lambda_{N}\right)$ and the Poisson bracket is defined by

$$
\begin{aligned}
\{f, g\}=\sum_{j=1}^{N}\left(\frac{\partial f}{\partial \phi_{1 j}} \frac{\partial g}{\partial \phi_{3 j}}+\frac{\partial f}{\partial \phi_{2 j}} \frac{\partial g}{\partial \phi_{4 j}}-\frac{\partial f}{\partial \phi_{3 j}} \frac{\partial g}{\partial \phi_{1 j}}\right. \\
\left.-\frac{\partial f}{\partial \phi_{4 j}} \frac{\partial g}{\partial \phi_{2 j}}+\frac{\partial f}{\partial \phi_{5 j}} \frac{\partial g}{\partial \phi_{5 j}}+\frac{\partial f}{\partial \phi_{6 j}} \frac{\partial g}{\partial \phi_{6 j}}\right) .
\end{aligned}
$$

Analogously, making use of (31) and (32), the $t_{2}$-part (27) of the two-component AKNS equations (24) is constrained as the following $6 \mathrm{~N}$-dimensional system:

$$
\begin{aligned}
& \phi_{1 j, t_{2}}=\left(-\lambda_{j}^{2}+\frac{1}{2} \widetilde{q}_{1} \widetilde{r}_{1}+\widetilde{\alpha}_{1} \widetilde{\beta}_{1}\right) \phi_{1 j}+\left(\tilde{q}_{1} \lambda_{j}-\frac{1}{2} \widetilde{q}_{1, x}\right) \phi_{3 j} \\
& +\left(\tilde{\alpha}_{1} \lambda_{j}-\tilde{\alpha}_{1, x}\right) \phi_{5 j}, \quad 1 \leq j \leq N, \\
& \phi_{2 j, t_{2}}=\left(-\lambda_{j}^{2}+\frac{1}{2} \widetilde{q}_{2} \widetilde{r}_{2}+\widetilde{\alpha}_{2} \widetilde{\beta}_{2}\right) \phi_{2 j}+\left(\tilde{q}_{2} \lambda_{j}-\frac{1}{2} \widetilde{q}_{2, x}\right) \phi_{4 j} \\
& +\left(\widetilde{\alpha}_{2} \lambda_{j}-\widetilde{\alpha}_{2, x}\right) \phi_{6 j}, \quad 1 \leq j \leq N, \\
& \phi_{3 j, t_{2}}=\left(\tilde{r}_{1} \lambda_{j}+\frac{1}{2} \widetilde{r}_{1, x}\right) \phi_{1 j}+\left(\lambda_{j}^{2}-\frac{1}{2} \widetilde{q}_{1} \widetilde{r}_{1}-\widetilde{\alpha}_{1} \widetilde{\beta}_{1}\right) \phi_{3 j} \\
& +\left(\widetilde{\beta}_{1} \lambda_{j}+\widetilde{\beta}_{1, x}\right) \phi_{5 j}, \quad 1 \leq j \leq N, \\
& \phi_{4 j, t_{2}}=\left(\widetilde{r}_{2} \lambda_{j}+\frac{1}{2} \widetilde{r}_{2, x}\right) \phi_{2 j}+\left(\lambda_{j}^{2}-\frac{1}{2} \widetilde{q}_{2} \widetilde{r}_{2}-\widetilde{\alpha}_{2} \widetilde{\beta}_{2}\right) \phi_{4 j} \\
& +\left(\widetilde{\beta}_{2} \lambda_{j}+\widetilde{\beta}_{2, x}\right) \phi_{6 j}, \quad 1 \leq j \leq N, \\
& \phi_{5 j, t_{2}}=\left(\widetilde{\beta}_{1} \lambda_{j}+\widetilde{\beta}_{1, x}\right) \phi_{1 j}+\left(-\widetilde{\alpha}_{1} \lambda_{j}+\widetilde{\alpha}_{1, x}\right) \phi_{3 j} \\
& 1 \leq j \leq N, \\
& \phi_{6 j, t_{2}}=\left(\widetilde{\beta}_{2} \lambda_{j}+\widetilde{\beta}_{2, x}\right) \phi_{2 j}+\left(-\widetilde{\alpha}_{2} \lambda_{j}+\widetilde{\alpha}_{2, x}\right) \phi_{4 j}, \\
& 1 \leq j \leq N
\end{aligned}
$$

where $\tilde{q}_{k}, \widetilde{r}_{k}, \widetilde{\alpha}_{k}, \widetilde{\beta}_{k}$ denote the constrained $q_{k}, r_{k}, \alpha_{k}, \beta_{k}$ and $\tilde{q}_{k, x}, \widetilde{r}_{k, x}, \widetilde{\alpha}_{k, x}, \widetilde{\beta}_{k, x}$ are given as follows:

$$
\begin{aligned}
& \tilde{q}_{1, x}=2\left\langle\Lambda \Phi_{1}, \Phi_{1}\right\rangle+2\left\langle\Phi_{1}, \Phi_{1}\right\rangle\left\langle\Phi_{1}, \Phi_{3}\right\rangle, \\
& \tilde{q}_{2, x}=2\left\langle\Lambda \Phi_{2}, \Phi_{2}\right\rangle+2\left\langle\Phi_{2}, \Phi_{2}\right\rangle\left\langle\Phi_{2}, \Phi_{4}\right\rangle, \\
& \tilde{r}_{1, x}=2\left\langle\Lambda \Phi_{3}, \Phi_{3}\right\rangle+2\left\langle\Phi_{3}, \Phi_{3}\right\rangle\left\langle\Phi_{1}, \Phi_{3}\right\rangle, \\
& \tilde{r}_{2, x}=2\left\langle\Lambda \Phi_{4}, \Phi_{4}\right\rangle+2\left\langle\Phi_{4}, \Phi_{4}\right\rangle\left\langle\Phi_{2}, \Phi_{4}\right\rangle, \\
& \tilde{\alpha}_{1, x}=-\left\langle\Lambda \Phi_{1}, \Phi_{5}\right\rangle-\left\langle\Phi_{1}, \Phi_{3}\right\rangle\left\langle\Phi_{1}, \Phi_{5}\right\rangle, \\
& \widetilde{\alpha}_{2, x}=-\left\langle\Lambda \Phi_{2}, \Phi_{6}\right\rangle-\left\langle\Phi_{2}, \Phi_{4}\right\rangle\left\langle\Phi_{2}, \Phi_{6}\right\rangle, \\
& \widetilde{\beta}_{1, x}=\left\langle\Lambda \Phi_{3}, \Phi_{5}\right\rangle+\left\langle\Phi_{1}, \Phi_{3}\right\rangle\left\langle\Phi_{3}, \Phi_{5}\right\rangle, \\
& \tilde{\beta}_{2, x}=\left\langle\Lambda \Phi_{4}, \Phi_{6}\right\rangle+\left\langle\Phi_{2}, \Phi_{4}\right\rangle\left\langle\Phi_{4}, \Phi_{6}\right\rangle .
\end{aligned}
$$

After a direct calculation, constrained system (35) can be written as the vector form

$$
\begin{array}{lll}
\Phi_{1, t_{2}}=\frac{\partial H_{2}}{\partial \Phi_{3}}, & \Phi_{2, t_{2}}=\frac{\partial H_{2}}{\partial \Phi_{4}}, & \Phi_{3, t_{2}}=-\frac{\partial H_{2}}{\partial \Phi_{1}}, \\
\Phi_{4, t_{2}}=-\frac{\partial H_{2}}{\partial \Phi_{2}}, & \Phi_{5, t_{2}}=\frac{\partial H_{2}}{\partial \Phi_{5}}, & \Phi_{6, t_{2}}=\frac{\partial H_{2}}{\partial \Phi_{6}},
\end{array}
$$

where

$$
\begin{aligned}
H_{2}= & -\left\langle\Lambda^{2} \Phi_{1}, \Phi_{3}\right\rangle-\left\langle\Lambda^{2} \Phi_{2}, \Phi_{4}\right\rangle-\frac{1}{2}\left\langle\Phi_{1}, \Phi_{1}\right\rangle\left\langle\Lambda \Phi_{3}, \Phi_{3}\right\rangle \\
& -\frac{1}{2}\left\langle\Phi_{2}, \Phi_{2}\right\rangle\left\langle\Lambda \Phi_{4}, \Phi_{4}\right\rangle-\frac{1}{2}\left\langle\Lambda \Phi_{1}, \Phi_{1}\right\rangle\left\langle\Phi_{3}, \Phi_{3}\right\rangle \\
& -\frac{1}{2}\left\langle\Lambda \Phi_{2}, \Phi_{2}\right\rangle\left\langle\Phi_{4}, \Phi_{4}\right\rangle \\
& -\frac{1}{2}\left\langle\Phi_{1}, \Phi_{1}\right\rangle\left\langle\Phi_{1}, \Phi_{3}\right\rangle\left\langle\Phi_{3}, \Phi_{3}\right\rangle \\
& -\frac{1}{2}\left\langle\Phi_{2}, \Phi_{2}\right\rangle\left\langle\Phi_{2}, \Phi_{4}\right\rangle\left\langle\Phi_{4}, \Phi_{4}\right\rangle \\
& +\left\langle\Phi_{1}, \Phi_{5}\right\rangle\left\langle\Lambda \Phi_{3}, \Phi_{5}\right\rangle \\
& +\left\langle\Phi_{2}, \Phi_{6}\right\rangle\left\langle\Lambda \Phi_{4}, \Phi_{6}\right\rangle+\left\langle\Lambda \Phi_{1}, \Phi_{5}\right\rangle\left\langle\Phi_{3}, \Phi_{5}\right\rangle \\
& +\left\langle\Lambda \Phi_{2}, \Phi_{6}\right\rangle\left\langle\Phi_{4}, \Phi_{6}\right\rangle+\left\langle\Phi_{1}, \Phi_{3}\right\rangle\left\langle\Phi_{1}, \Phi_{5}\right\rangle\left\langle\Phi_{3}, \Phi_{5}\right\rangle \\
& +\left\langle\Phi_{2}, \Phi_{4}\right\rangle\left\langle\Phi_{2}, \Phi_{6}\right\rangle\left\langle\Phi_{4}, \Phi_{6}\right\rangle .
\end{aligned}
$$

To this end, we show that the constrained $N$ copies of systems (7) and (27) are super Hamiltonian systems (32) and (37).

\section{4. $r$-Matrix and Lax Representation}

In what follows, we will show that super Hamiltonian systems (32) and (37) are both completely integrable in the Liouville sense. First of all, through a straightforward and tedious calculation, we arrive at the following proposition. 
Proposition 1. Super Hamiltonian systems (32) and (37) have the following Lax representations, respectively:

$$
\begin{aligned}
L_{x}(\lambda) & =[\widetilde{U}, L(\lambda)], \\
L_{t_{2}}(\lambda) & =\left[\widetilde{V}^{(2)}, L(\lambda)\right],
\end{aligned}
$$

where $\widetilde{U}$ and $\widetilde{V}^{(2)}$ are, respectively, $U$ and $V^{(2)}$ under symmetry constraint (31), and

$$
L(\lambda)=\left(\begin{array}{cccccc}
A_{1}(\lambda) & 0 & B_{1}(\lambda) & 0 & \varrho_{1}(\lambda) & 0 \\
0 & A_{2}(\lambda) & 0 & B_{2}(\lambda) & 0 & \varrho_{2}(\lambda) \\
C_{1}(\lambda) & 0 & -A_{1}(\lambda) & 0 & \omega_{1}(\lambda) & 0 \\
0 & C_{2}(\lambda) & 0 & -A_{2}(\lambda) & 0 & \omega_{2}(\lambda) \\
\omega_{1}(\lambda) & 0 & -\varrho_{1}(\lambda) & 0 & 0 & 0 \\
0 & \omega_{2}(\lambda) & 0 & -\varrho_{2}(\lambda) & 0 & 0
\end{array}\right)
$$

with

$$
\begin{aligned}
& A_{1}(\lambda)=-1+\sum_{j=1}^{N} \frac{1}{\lambda-\lambda_{j}} \phi_{1 j} \phi_{3 j}, \\
& A_{2}(\lambda)=-1+\sum_{j=1}^{N} \frac{1}{\lambda-\lambda_{j}} \phi_{2 j} \phi_{4 j}, \\
& B_{1}(\lambda)=-\sum_{j=1}^{N} \frac{1}{\lambda-\lambda_{j}} \phi_{1 j}^{2} \\
& B_{2}(\lambda)=-\sum_{j=1}^{N} \frac{1}{\lambda-\lambda_{j}} \phi_{2 j}^{2} \\
& C_{1}(\lambda)=\sum_{j=1}^{N} \frac{1}{\lambda-\lambda_{j}} \phi_{3 j}^{2} \\
& C_{2}(\lambda)=\sum_{j=1}^{N} \frac{1}{\lambda-\lambda_{j}} \phi_{4 j}^{2} \\
& \varrho_{1}(\lambda)=\sum_{j=1}^{N} \frac{1}{\lambda-\lambda_{j}} \phi_{1 j} \phi_{5 j}, \\
& \varrho_{2}(\lambda)=\sum_{j=1}^{N} \frac{1}{\lambda-\lambda_{j}} \phi_{2 j} \phi_{6 j}, \\
& \omega_{1}(\lambda)=\sum_{j=1}^{N} \frac{1}{\lambda-\lambda_{j}} \phi_{3 j} \phi_{5 j}, \\
& \omega_{2}(\lambda)=\sum_{j=1}^{N} \frac{1}{\lambda-\lambda_{j}} \phi_{4 j} \phi_{6 j} .
\end{aligned}
$$

Under the Poisson bracket (34), we have

$$
\begin{aligned}
& \left\{A_{k}(\lambda), B_{k}(\lambda)\right\}=\frac{2}{\mu-\lambda}\left(-B_{k}(\lambda)+B_{k}(\mu)\right) \\
& =-\left\{B_{k}(\lambda), A_{k}(\lambda)\right\}, \quad k=1,2, \\
& \left\{A_{k}(\lambda), C_{k}(\lambda)\right\}=\frac{2}{\mu-\lambda}\left(C_{k}(\lambda)-C_{k}(\mu)\right) \\
& =-\left\{C_{k}(\lambda), A_{k}(\lambda)\right\}, \quad k=1,2, \\
& \left\{A_{k}(\lambda), \varrho_{k}(\lambda)\right\}=\frac{1}{\mu-\lambda}\left(-\varrho_{k}(\lambda)+\varrho_{k}(\mu)\right) \\
& =-\left\{\varrho_{k}(\lambda), A_{k}(\lambda)\right\}, \quad k=1,2, \\
& \left\{A_{k}(\lambda), \omega_{k}(\lambda)\right\}=\frac{1}{\mu-\lambda}\left(\omega_{k}(\lambda)-\omega_{k}(\mu)\right) \\
& =-\left\{\omega_{k}(\lambda), A_{k}(\lambda)\right\}, \quad k=1,2, \\
& \left\{B_{k}(\lambda), C_{k}(\lambda)\right\}=\frac{4}{\mu-\lambda}\left(-A_{k}(\lambda)+A_{k}(\mu)\right) \\
& =-\left\{C_{k}(\lambda), B_{k}(\lambda)\right\}, \quad k=1,2, \\
& \left\{B_{k}(\lambda), \omega_{k}(\lambda)\right\}=\frac{2}{\mu-\lambda}\left(-\varrho_{k}(\lambda)+\varrho_{k}(\mu)\right) \\
& =-\left\{\omega_{k}(\lambda), B_{k}(\lambda)\right\}, \quad k=1,2, \\
& \left\{C_{k}(\lambda), \varrho_{k}(\lambda)\right\}=\frac{2}{\mu-\lambda}\left(-\omega_{k}(\lambda)+\omega_{k}(\mu)\right) \\
& =-\left\{\varrho_{k}(\lambda), C_{k}(\lambda)\right\}, \quad k=1,2, \\
& \left\{\varrho_{k}(\lambda), \varrho_{k}(\lambda)\right\}=\frac{1}{\mu-\lambda}\left(-B_{k}(\lambda)+B_{k}(\mu)\right), \quad k=1,2 \text {, } \\
& \left\{\omega_{k}(\lambda), \omega_{k}(\lambda)\right\}=\frac{1}{\mu-\lambda}\left(C_{k}(\lambda)-C_{k}(\mu)\right), \quad k=1,2, \\
& \left\{\varrho_{k}(\lambda), \omega_{k}(\lambda)\right\}=\frac{1}{\mu-\lambda}\left(A_{k}(\lambda)-A_{k}(\mu)\right) \\
& =\left\{\omega_{k}(\lambda), \varrho_{k}(\lambda)\right\}, \quad k=1,2,
\end{aligned}
$$

and the others are zero.

These relations imply the following proposition immediately.

Proposition 2. The Lax matrix $L(\lambda)$ satisfied the following $r$ matrix relation:

$$
\left\{L_{1}(\lambda), L_{2}(\mu)\right\}=\frac{1}{\mu-\lambda}\left[P, L_{1}(\lambda)+L_{2}(\mu)\right],
$$

where

$$
\begin{aligned}
P= & \sigma_{1} \otimes \sigma_{1}+\sigma_{2} \otimes \sigma_{2} \\
& +2\left(\sigma_{3} \otimes \sigma_{5}+\sigma_{5} \otimes \sigma_{3}+\sigma_{4} \otimes \sigma_{6}+\sigma_{6} \otimes \sigma_{4}\right) \\
& +\sigma_{7} \otimes \sigma_{9}+\sigma_{9} \otimes \sigma_{7}+\sigma_{8} \otimes \sigma_{10}+\sigma_{10} \otimes \sigma_{8}
\end{aligned}
$$


with

$$
\begin{array}{ccc}
\sigma_{1}=E_{11}-E_{33}, & \sigma_{2}=E_{22}-E_{44}, \quad \sigma_{3}=E_{13}, \\
\sigma_{4}=E_{24}, & \sigma_{5}=E_{31}, \quad \sigma_{6}=E_{42}, \\
\sigma_{7}=E_{15}-E_{53}, \quad \sigma_{8}=E_{26}-E_{64}, & \sigma_{9}=E_{35}+E_{51}, \\
\sigma_{10}=E_{46}+E_{62}, &
\end{array}
$$

$E_{i j}$ is the $6 \times 6$ matrix having 1 in the $(i, j)$ th position and zeros elsewhere, and $L_{1}(\lambda)=L(\lambda) \otimes I$ and $L_{2}(\mu)=I \otimes L(\mu)$.

Therefore, according to the general theory of the $r$-matrix [22], we know that $(1 / 2) \operatorname{Str} L^{2}(\lambda)$ is a generating function of conserved integrals of motions. Explicitly, we can expand $(1 / 2) \operatorname{Str} L^{2}(\lambda)$ as follows:

$$
\frac{1}{2} \operatorname{Str} L^{2}(\lambda)=\sum_{n \geq 0} F_{n} \lambda^{-n}
$$

where

$$
\begin{aligned}
F_{1}=-2\left\langle\Phi_{1}, \Phi_{3}\right\rangle-2\left\langle\Phi_{2}, \Phi_{4}\right\rangle \\
F_{n}=\sum_{j=1}^{n-1}\left[\left\langle\Lambda^{j-1} \Phi_{1}, \Phi_{3}\right\rangle\left\langle\Lambda^{n-j-1} \Phi_{1}, \Phi_{3}\right\rangle\right. \\
-\left\langle\Lambda^{j-1} \Phi_{1}, \Phi_{1}\right\rangle\left\langle\Lambda^{n-j-1} \Phi_{3}, \Phi_{3}\right\rangle \\
+2\left\langle\Lambda^{j-1} \Phi_{1}, \Phi_{5}\right\rangle\left\langle\Lambda^{n-j-1} \Phi_{3}, \Phi_{5}\right\rangle \\
+\left\langle\Lambda^{j-1} \Phi_{2}, \Phi_{4}\right\rangle\left\langle\Lambda^{n-j-1} \Phi_{2}, \Phi_{4}\right\rangle \\
-\left\langle\Lambda^{j-1} \Phi_{2}, \Phi_{2}\right\rangle\left\langle\Lambda^{n-j-1} \Phi_{4}, \Phi_{4}\right\rangle \\
\left.+2\left\langle\Lambda^{j-1} \Phi_{2}, \Phi_{6}\right\rangle\left\langle\Lambda^{n-j-1} \Phi_{4}, \Phi_{6}\right\rangle\right] \\
-2\left\langle\Lambda^{n-1} \Phi_{1}, \Phi_{3}\right\rangle-2\left\langle\Lambda^{n-1} \Phi_{2}, \Phi_{4}\right\rangle, \quad n \geq 2 .
\end{aligned}
$$

Therefore, from (44), we have

$$
\left\{F_{m}, F_{n}\right\}=0, \quad 1 \leq m, n \leq 3 N
$$

which means that $\left\{F_{n}\right\}_{n=1}^{3 N}$ are in involution. Moreover, referring to proof of functional independence in [15], it is easy to find that $\left\{F_{n}\right\}_{n=1}^{3 N}$ are functionally independent over some region of $\mathbb{R}^{6 N}$.

To sum up the above results, we have the following theorem.

Theorem 3. The super Hamiltonian systems given by (32) and (37) are both completely integrable in the sense of Liouville. That is to say, Hamiltonian systems (32) and (37) constitute an integrable decomposition of two-component super AKNS equations (26).

\section{Conclusions and Discussions}

Starting from a matrix Lie superalgebra, we constructed a two-component super AKNS system (19). For this new system, we considered a Bargmann symmetry constraint (31). Then, substituting the constraint (31) into $N$ copies of systems (7) and (27), we obtained the constrained super Hamiltonian systems (32) and (37), and we showed that systems (32) and (37) are completely integrable in the Liouville sense. Accordingly, Lax matrix $L(\lambda)$ and $r$-matrix representation were, respectively, given in Propositions 1 and 2. The difference between [15] and this paper will be listed in the following.

(1) In [15], we applied binary-nonlinearization to onecomponent AKNS system which was associated with a $3 \times 3$ spectral matrix, while, in this paper, we applied monononlinearization to two-component AKNS system which was associated with a $6 \times 6$ spectral matrix.

(2) In [15], symmetry constraint was associated with both eigenfunctions and adjoint eigenfunctions, while, in this paper, constraint was just associated with eigenfunctions.

(3) Construction of integrals of motion is also different. In [15], we made use of constrained stationary equation $\left(\widetilde{N}^{2}\right)_{x}=\left[\widetilde{M}, \widetilde{N}^{2}\right]$. And, in this paper, we made use of general theory of $r$-matrix.

According to the above conclusions, some future work is listed as follows.

(1) Is this method applied to the other multicomponent super integrable system?

(2) If potentials $q$ and $r$ are chosen as (2) in [23], is nonlinearization of Lax pairs applied to this multicomponent integrable system?

(3) Is nonlinearization (including monononlinearization and binary-nonlinearization) extended to supersymmetry integrable system, such as supersymmetric Kadomtsev-Petviashvili system [24, 25]?

\section{Conflict of Interests}

The authors declare that there is no conflict of interests regarding the publication of this paper.

\section{Acknowledgments}

This work is supported by the National Natural Science Foundation of China under Grant nos. 11001069 and 61273077 and Zhejiang Provincial Natural Science Foundation of China under Grant no. LQ12A01002.

\section{References}

[1] W. Ma and R. Zhou, "Adjoint symmetry constraints of multicomponent AKNS equations," Chinese Annals of Mathematics $B$, vol. 23, no. 3, pp. 373-384, 2002.

[2] Y. Zhang, "A multi-component matrix loop algebra and a unified expression of the multi-component AKNS hierarchy 
and the multi-component BPT hierarchy," Physics Letters A: General, Atomic and Solid State Physics, vol. 342, no. 1-2, pp. 8289, 2005.

[3] A. P. Fordy and P. P. Kulish, "Nonlinear Schrödinger equations and simple Lie algebras," Communications in Mathematical Physics, vol. 89, no. 3, pp. 427-443, 1983.

[4] T. Tsuchida and M. Wadati, "The coupled modified Kortewegde vries equations," Journal of the Physical Society of Japan, vol. 67, no. 4, pp. 1175-1187, 1998.

[5] C. W. Cao, "A cubic system which generates Bargmann potential and N-gap potential," Chinese Quarterly Journal of Mathematics, vol. 3, no. 1, pp. 90-96, 1988.

[6] Y. Zeng and Y. Li, "The constraints of potentials and the finite-dimensional integrable systems," Journal of Mathematical Physics, vol. 30, no. 8, pp. 1679-1689, 1989.

[7] C. W. Cao, "Nonlinearization of the Lax system for AKNS hierarchy," Science in China A: Mathematics, Physics, Astronomy, vol. 33, no. 5, pp. 528-536, 1990.

[8] C. Cewen and G. Xianguo, "C Neumann and Bargmann systems associated with the coupled KdV soliton hierarchy," Journal of Physics A: Mathematical and General, vol. 23, no. 18, pp. 41174125, 1990.

[9] R. Zhou, "Dynamical r-matrices for the constrained HarryDym flows," Physics Letters A: General, Atomic and Solid State Physics, vol. 220, no. 6, pp. 320-330, 1996.

[10] Y. Zeng and J. Hietarinta, "Classical poisson structures and rmatrices from constrained flows," Journal of Physics A: Mathematical and General, vol. 29, no. 16, pp. 5241-5252, 1996.

[11] W.-X. Ma and W. Strampp, "An explicit symmetry constraint for the Lax pairs and the adjoint Lax pairs of AKNS systems," Physics Letters A, vol. 185, no. 3, pp. 277-286, 1994.

[12] W. X. Ma, "Symmetry constraint of MKdV equations by binary nonlinearization," Physica A: Statistical Mechanics and its Applications, vol. 219, no. 3-4, pp. 467-481, 1995.

[13] W.-X. Ma and R. Zhou, "Adjoint symmetry constraints leading to binary nonlinearization," Journal of Nonlinear Mathematical Physics, vol. 9, supplement 1, pp. 106-126, 2002.

[14] J. Ji and R. Zhou, "Two types of new integrable decompositions of the Kaup-Newell equation," Chaos, Solitons \& Fractals, vol. 30, no. 4, pp. 993-1003, 2006.

[15] J. He, J. Yu, Y. Cheng, and R. Zhou, "Binary nonlinearization of the super AKNS system," Modern Physics Letters B, vol. 22, no. 4, pp. 275-288, 2008.

[16] J. Yu, J. He, W. Ma, and Y. Cheng, “The Bargmann symmetry constraint and binary nonlinearization of the super Dirac systems," Chinese Annals of Mathematics B, vol. 31, no. 3, pp. 361-372, 2010.

[17] J. Yu, J. He, Y. Cheng, and J. Han, "A novel symmetry constraint of the super cKdV system," Journal of Physics A: Mathematical and Theoretical, vol. 43, no. 44, Article ID 445201, 12 pages, 2010.

[18] L. Yi-Shen and Z. Li-ning, "Super AKNS scheme and its infinite conserved currents," Il Nuovo Cimento A, vol. 93, no. 2, pp. 175$183,1986$.

[19] L. Yi-shen and Z. Li-ning, "A note on the super AKNS equations," Journal of Physics A: Mathematical and General, vol. 21, no. 7, pp. 1549-1552, 1988.

[20] X.-B. Hu, "An approach to generate superextensions of integrable systems," Journal of Physics A: Mathematical and General, vol. 30, no. 2, pp. 619-632, 1997.

[21] W.-X. Ma, J.-S. He, and Z.-Y. Qin, "A supertrace identity and its applications to superintegrable systems," Journal of
Mathematical Physics, vol. 49, no. 3, Article ID 033511, 13 pages, 2008.

[22] O. Babelon and C.-M. Viallet, "Hamiltonian structures and Lax equations," Physics Letters B: Nuclear, Elementary Particle and High-Energy Physics, vol. 237, no. 3-4, pp. 411-416, 1990.

[23] D.-S. Wang, D.-J. Zhang, and J. Yang, "Integrable properties of the general coupled nonlinear Schrödinger equations," Journal of Mathematical Physics, vol. 51, no. 2, Article ID 023510, 2010.

[24] Yu. I. Manin and A. O. Radul, "A supersymmetric extension of the Kadomtsev-Petviashvili hierarchy," Communications in Mathematical Physics, vol. 98, no. 1, pp. 65-77, 1985.

[25] J. He and Y. Cheng, "The KW theorem for the SKP hierarchy," Physics Letters B: Nuclear, Elementary Particle and High-Energy Physics, vol. 449, no. 3-4, pp. 194-200, 1999. 


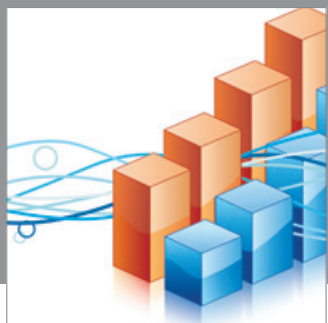

Advances in

Operations Research

mansans

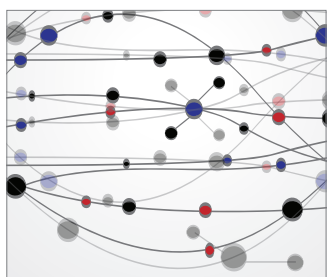

The Scientific World Journal
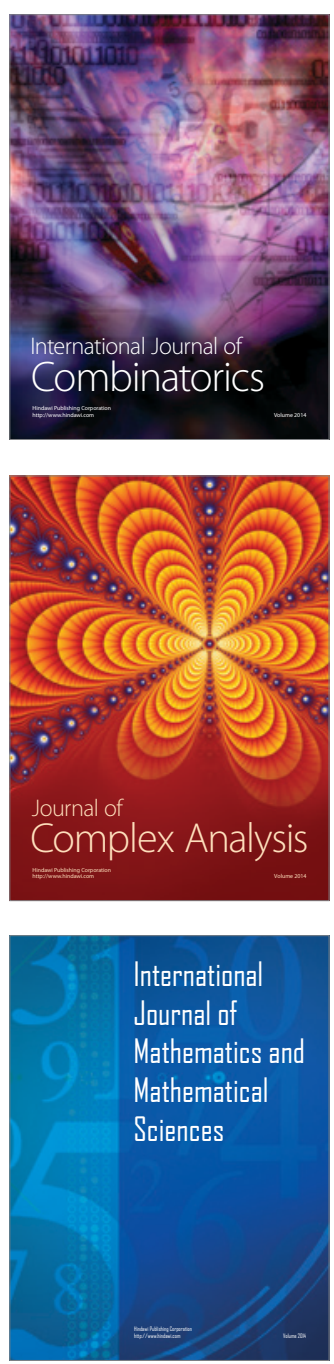
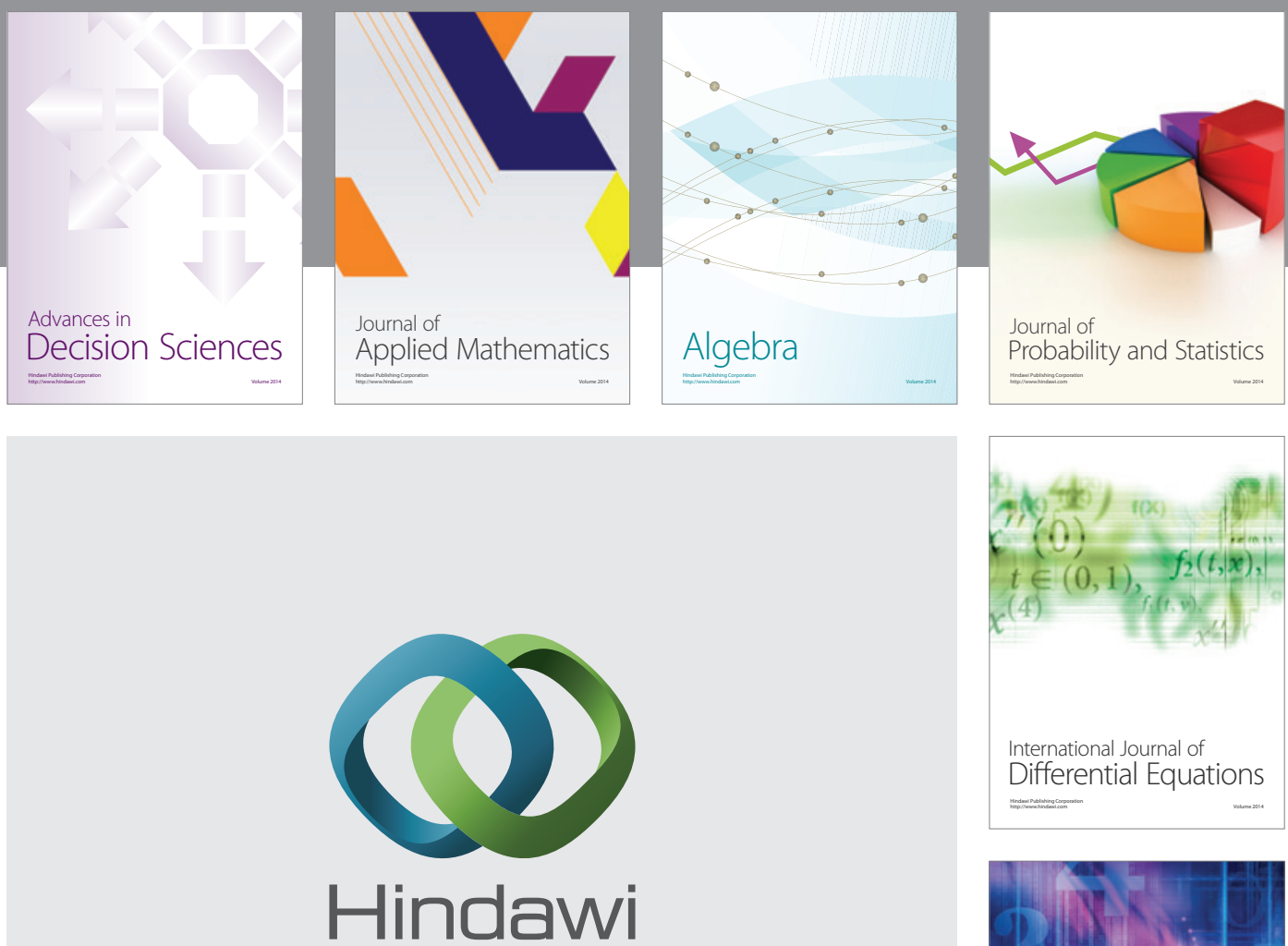

Submit your manuscripts at http://www.hindawi.com
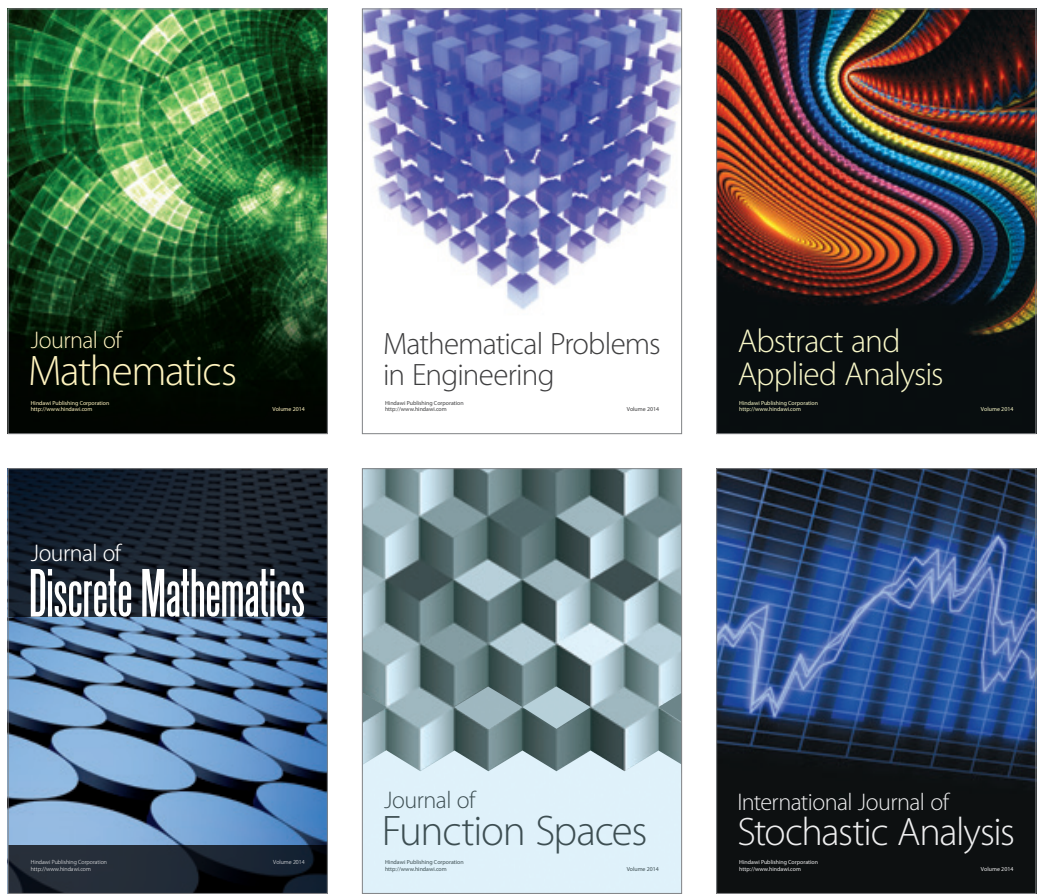

Journal of

Function Spaces

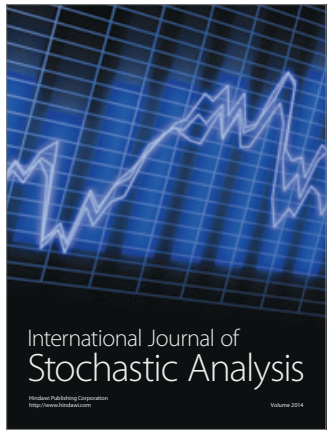

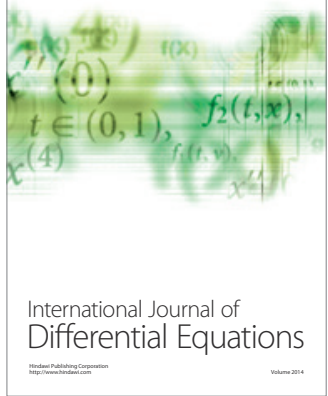
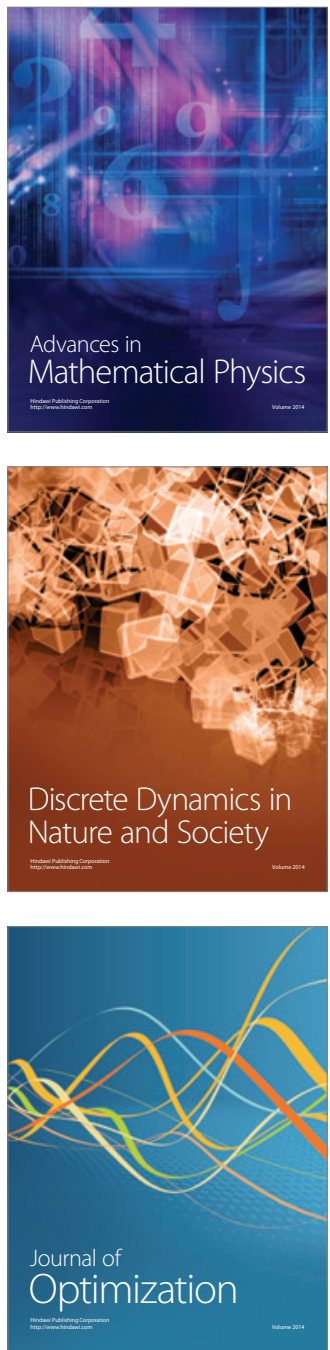